\title{
MAP LATTICES COUPLED BY COLLISIONS
}

\author{
GERHARD KELLER AND CARLANGELO LIVERANI
}

\begin{abstract}
We introduce a new coupled map lattice model in which the weak interaction takes place via rare "collisions". By "collision" we mean a strong (possibly discontinuous) change in the system. For such models we prove uniqueness of the SRB measure and exponential space-time decay of correlations.
\end{abstract}

\section{INTRODUCTION}

During the last years many results have been published on coupled map lattices. Much of this work deals with the weak coupling situation; see [3] and references therein to have a more precise idea of all the related work and results. In most of this considerable body of work the weak coupling is described by a diffeomorphism of the state space close to identity. Only recently couplings close to identity but not diffeomorphic could be investigated in a mathematically rigorous way. This setting (coupling close to identity) models a weak interaction and is reminiscent of a situation in which a collection of systems is interacting via a weak potential. Yet, a collection of systems can also have interactions that are weak only on average. The typical example of such a situation are interactions that can be strong but are rare, such as in the case of collisions in a rarefied gas. Examples of such a situation that has attracted some attention lately are models of the type introduced in [9] and recently used in $[5,6]$ to argue for a derivation of the Fourier Law from microscopic dynamics.

In this note we consider a simple system of coupled dynamics of the latter type. Namely a lattice of piecewise expanding interval maps with strong but rare interactions. Of course, this model is very far from a system of interacting disks, yet it is interesting that the available techniques can be applied to a case with strong but rare interactions. Given the present efforts in trying to devise a setting for Anosov maps with discontinuities that shares the same good properties of the $B V$ setting for piecewise expanding maps $[4,1]$ it is reasonable to hope that in the future the present results could be extend to more realistic situations.

What we prove is that such a system, if the local maps are sufficiently expanding, has a unique SRB measure with exponential mixing properties in time and space.

Date: November 4, 2018

2000 Mathematics Subject Classification. 37C30,37L60,37D20.

Key words and phrases. Coupled map lattice, SRB measure, exponential decay of correlations.

We are indebted to the ESI where, during the Workshop on Hyperbolic Dynamical Systems with Singularities (2008), this work was started. L.C. thanks the ENS, Paris, where he was invited during part of this work. Also we like to thank the Institut Henri Poincare - Centre Emile Borel where, during the trimester Mécanique statistique, probabilités et systèmes de particules (2008), this work was finished. Finally, G.K. acknowledges the support by a grant from the DFG. 
These are the same type of results we proved for systems with weak interactions of the "coupling close to identity" type in [8].

\section{The System And the Results}

Consider $X=[0,1]^{\mathbb{Z}^{d}}$ and a map $T:[0,1] \rightarrow[0,1]$, piecewise smooth and expanding, that is $|D T| \geq \lambda>1$. The uncoupled system is described by the product dynamics $F_{0}: X \rightarrow X$ defined by $F_{0}(x)_{i}=T\left(x_{i}\right)$. The strength of the interaction will be expressed by a parameter $\varepsilon>0$ which measures how rare the interactions are. To be precise, let $\left\{e_{i}\right\}$ be the standard base of $\mathbb{R}^{d}$ and $V:=\left\{e_{i},-e_{i}\right\} \subset \mathbb{R}^{d}$, and, for each $\varepsilon>0$ sufficiently small, fix a set of disjoint open intervals $\left\{A_{\varepsilon, v}\right\}_{v \in V} \subset[0,1]$ such that $\left|A_{\varepsilon, v}\right|=\varepsilon$. Consider the coupling ${ }^{1}$

$$
\left(\Phi_{\varepsilon}(x)\right)_{i}= \begin{cases}x_{i+v} & \text { if } x_{i} \in A_{\varepsilon, v} \text { and } x_{i+v} \in A_{\varepsilon,-v} \text { for some } v \in V \\ x_{i} & \text { otherwise. }\end{cases}
$$

The dynamics we are interested in is then defined by $F_{\varepsilon}=\Phi_{\varepsilon} \circ F_{0}$.

Remark 2.1. The interpretation of $\Phi_{\varepsilon}$ is quite obvious: nearby systems can interact only if the coordinates belong to a small set (the "collision" set). If this is the case, then the coordinates undergo a violent change.

We use $\|\cdot\|$ for the bounded variation norm and $|\cdot|$ for the total variation norm of a measure. That is, calling $\mathcal{D}$ the space of differentiable local functions on $X$ and $\mathcal{D}_{1}:=\left\{\varphi \in \mathcal{D}:|\varphi|_{\infty} \leq 1\right\}$, for each complex Borel measure $\mu$ on $X$ we define

$$
\begin{aligned}
|\mu| & :=\sup _{\varphi \in \mathcal{D}_{1}} \mu(\varphi) \\
\|\mu\| & :=\sup _{i \in \mathbb{Z}^{d}} \sup _{\varphi \in \mathcal{D}_{1}} \mu\left(\partial_{x_{i}} \varphi\right) .
\end{aligned}
$$

To study the statistical properties of the map $F_{\varepsilon}$ we will follow the well established path of studying its action on the space of measures. That is we will investigate the operator $F_{\varepsilon}^{*}{ }^{2}$ To this end we will follow the path laid down in [8] and restrict our study to measures belonging to the space $\mathcal{B}:=\{\|\mu\|<\infty\}$, see [7] for a detailed explanation of the meaning and properties of this choice. We will then use exactly the same strategy as developed in [8].

The following lemma is proven in [7, Proposition 4]; see also Lemma 2.2 of [8].

Lemma 2.2. There exists a constant $B_{0}>0$ such that for each $\mu \in \mathcal{B}$ holds

$$
\begin{aligned}
\left|F_{0}^{*} \mu\right| & \leq|\mu| \\
\left\|F_{0}^{*} \mu\right\| & \leq 2 \lambda^{-1}\|\mu\|+B_{0}|\mu| .
\end{aligned}
$$

The proof of the next lemma is provided in section 3.

Lemma 2.3. There exists a constant $B_{1}>0$ such that for each $\mu \in \mathcal{B}$ holds

$$
\begin{aligned}
\left|\Phi_{\varepsilon}^{*} \mu\right| & \leq|\mu| \\
\left\|\Phi_{\varepsilon}^{*} \mu\right\| & \leq(2+2 d)\|\mu\|+B_{1} \ell_{\varepsilon}^{-1}|\mu| .
\end{aligned}
$$

where $\ell_{\varepsilon}$ is the minimal distance between the intervals $A_{\varepsilon, v}(v \in V)$.

\footnotetext{
${ }^{1}$ This special case is considered for simplicity. Yet, one can easily treat the more general case in which $x_{i+v}$ is replaced by $\phi_{v}\left(x_{i}, x_{i+v}\right)$, for some set of invertible smooth maps $\phi_{v}:[0,1]^{2} \rightarrow[0,1]^{2}$.

${ }^{2}$ As usual for each map $\Psi: X \rightarrow X$ the operator $\Psi^{*}$ is defined by $\Psi^{*} \mu(\varphi)=\mu(\varphi \circ \Psi)$, for each measure $\mu$ and function $\varphi$.
} 
The above lemmas imply a Lasota-Yorke type inequality for $F_{\varepsilon}$, namely: there exists a constant $B>0$ such that

$$
\begin{aligned}
\left|F_{\varepsilon}^{*} \mu\right| & \leq|\mu| \\
\left\|F_{\varepsilon}^{*} \mu\right\| & \leq(4+4 d) \lambda^{-1}\|\mu\|+B \ell_{\varepsilon}^{-1}|\mu| .
\end{aligned}
$$

The second of these inequalities is relevant in the case $\sigma=(4+4 d) \lambda^{-1}<1$ which we will assume from now on. ${ }^{3}$ The only thing left is to define a one-site decoupling $\Phi_{\varepsilon, i}$ of the dynamics and to show that we can decouple at a single site paying only a small price. Namely, we define

$$
\left(\Phi_{\varepsilon, i}(x)\right)_{k}= \begin{cases}\left(\Phi_{\varepsilon}(x)\right)_{k} & \text { if } k-i \notin V \cup\{0\} \\ \left(\Phi_{\varepsilon}(x)\right)_{k} & \text { if } v:=k-i \in V, \text { and } x_{k} \notin A_{\varepsilon,-v} \\ x_{k} & \text { otherwise. }\end{cases}
$$

A moment of thought will show that $\Phi_{\varepsilon, i}$ differs from $\Phi_{\varepsilon}$ only insofar the coordinate $x_{i}$ is now independent of the other coordinates.

Lemma 2.4. For each $i \in \mathbb{Z}^{d}$,

$$
\begin{aligned}
\left|\Phi_{\varepsilon, i}^{*} \mu\right| & \leq|\mu| \\
\left\|\Phi_{\varepsilon, i}^{*} \mu\right\| & \leq(2+2 d)\|\mu\|+B_{1} \ell_{\varepsilon}^{-1}|\mu| \\
\left|\Phi_{\varepsilon}^{*} \mu-\Phi_{\varepsilon, i}^{*} \mu\right| & \leq 4 d \varepsilon\|\mu\| .
\end{aligned}
$$

The proof of this lemma can be found in Section 3.

Equation (2.2) and Lemma 2.4 are all that is needed to prove our main theorem.

Theorem 2.5. If $\lambda>4+4 d$ and if $\liminf _{\varepsilon \backslash 0} \ell_{\varepsilon}>0$, then there is some $\varepsilon_{0}>0$ such that for each $\varepsilon \in\left(0, \varepsilon_{0}\right)$ there exists a unique invariant element $\mu_{i n v} \in \mathcal{B}$ for the dynamics $F_{\varepsilon}$. In addition, for all local smooth observables, $\mu_{i n v}$ enjoys exponential decay of space-time correlations.

Proof. The proof follows verbatim the arguments in [8]. More precisely, Lemma 2.3 of [8] holds in the present context under the above hypotheses. The proof of such a lemma is exactly the same as in Section 3 of [8], provided:

a) Lemma 2.2 of [8] is replaced by inequalities (2.2) above;

b) instead of inequality (3.16) of [8] (and of Lemma 3.2 in [8] from which that estimate follows) one uses the last assertion of our Lemma 2.4;

c) the analog of equation (3.17) in [8] is now obtained using estimate (2.2) above instead of Lemma 2.2 of [8].

The proof of the theorem is then exactly the same as the proof of Theorem 2.1 of [8] (see section 4 of the cited paper).

\section{Proofs}

Proof of Lemma 2.3. Let $\varphi$ be a smooth local function and let $i \in \mathbb{Z}^{d}$. Then, setting $\Delta_{\varepsilon, v, i}=\left\{x: x_{i} \in A_{\varepsilon, v}, x_{i+v} \in A_{\varepsilon,-v}\right\}$ and $\Delta_{\varepsilon, i}=\cup_{v \in V} \Delta_{\varepsilon, v, i}$, we have

$$
\left(\partial_{x_{i}} \varphi\right) \circ \Phi_{\varepsilon}(x)= \begin{cases}\partial_{x_{i}}\left(\left(\varphi \circ \Phi_{\varepsilon}\right)\right)(x) & \text { if } x \in \Delta_{\varepsilon, i}^{c} \\ \partial_{x_{i+v}}\left(\left(\varphi \circ \Phi_{\varepsilon}\right)\right)(x) & \text { if } x \in \Delta_{\varepsilon, v, i} .\end{cases}
$$

\footnotetext{
${ }^{3}$ With some more work one can certainly weaken the requirement on the expansion constant $\lambda$. Since the goal of this note is to show that this type of maps can be treated by transfer operator methods, we decided to restrict ourselves to the simplest possible setting.
} 
Therefore, for $\mu \in \mathcal{B}$,

$$
\begin{aligned}
\Phi_{\varepsilon}^{*} \mu\left(\partial_{x_{i}} \varphi\right) & =\int_{X}\left(\partial_{x_{i}} \varphi\right) \circ \Phi_{\varepsilon} d \mu \\
& =\int_{\Delta_{\varepsilon, i}^{c}} \partial_{x_{i}}\left(\varphi \circ \Phi_{\varepsilon}\right) d \mu+\sum_{v \in V} \int_{\Delta_{\varepsilon, v, i}} \partial_{x_{i+v}}\left(\varphi \circ \Phi_{\varepsilon}\right) d \mu .
\end{aligned}
$$

In order to estimate these integrals against the variation of $\mu$ in directions $i$ and $i+v$ we must modify the test function $\varphi \circ \Phi_{\varepsilon}$ in such a way that it becomes continuous in $x_{i}$ or $x_{i+v}$, respectively (see the characterization of $\|\cdot\|$ in [7] section 3.3).

For the first integral, let $\varphi_{*}$ be a function that, for each fixed $x_{\neq i}$, is piecewise linear (but not necessarily continuous!) in $x_{i}$ interpolating between the requirements $\left.\varphi_{*}\right|_{\Delta_{\varepsilon, i}}=0$ and $\varphi_{*}(x)=\varphi\left(\Phi_{\varepsilon}(x)\right)$ for each $x=\left(x_{\neq i}, x_{i}\right) \in \partial_{i} \Delta_{\varepsilon, i}$ where $\partial_{i} \Delta_{\varepsilon, i}$ denotes the set of those points in the boundary of $\Delta_{\varepsilon, i}$ where the boundary is normal to the $x_{i}$-direction. Thus, given $x_{\neq i}$, the partial derivative $\partial_{x_{i}} \varphi_{*}(x)$ exists for Lebesgue-a.e. $x_{i}$, and $\tilde{\varphi}:=1_{\Delta_{\varepsilon, i}^{c}} \cdot\left(\varphi \circ \Phi_{\varepsilon}\right)-\varphi_{*}$ is Lipschitz continuous in $x_{i}$, $|\tilde{\varphi}|_{\infty} \leq 2|\varphi|_{\infty},\left.\tilde{\varphi}\right|_{\partial_{i} \Delta_{\varepsilon, i}}=0$, and $\left|\partial_{x_{i}} \varphi_{*}\right|_{\infty} \leq C \ell_{\varepsilon}^{-1}|\varphi|_{\infty}$ where $\ell_{\varepsilon}$ is the minimal distance between intervals $A_{\varepsilon, v}(v \in V)$. Hence,

$$
\int_{\Delta_{\varepsilon, i}^{c}} \partial_{x_{i}}\left(\varphi \circ \Phi_{\varepsilon}\right) d \mu=\int_{X} \partial_{x_{i}} \tilde{\varphi} d \mu+\int_{X} \partial_{x_{i}} \varphi_{*} d \mu \leq 2|\varphi|_{\infty}\|\mu\|+C \ell_{\varepsilon}^{-1}|\varphi|_{\infty}|\mu| .
$$

Similarly, for each $v \in V$, let $\varphi_{v}$ be a function that, for each fixed $x_{\neq i+v}$, is piecewise constant in $x_{i+v}$ and such that $\left.\varphi_{v}\right|_{\Delta_{\varepsilon, i, v}}=0$ and $\varphi_{v}(x)=\varphi\left(\Phi_{\varepsilon}(x)\right)$ for each $x=\left(x_{\neq i+v}, x_{i+v}\right) \in \partial_{i+v} \Delta_{\varepsilon, i, v}$. Then $\tilde{\varphi}_{v}:=1_{\Delta_{\varepsilon, i, v}} \cdot\left(\varphi \circ \Phi_{\varepsilon}\right)+\varphi_{v}$ is Lipschitz continuous in $x_{i+v},\left|\tilde{\varphi}_{v}\right|_{\infty} \leq|\varphi|_{\infty}$ and $\partial_{x_{i+v}} \varphi_{v}(x)=0$ for $x \notin \partial_{i+v} \Delta_{\varepsilon, i, v}$. Hence

$$
\int_{\Delta_{\varepsilon, v, i}} \partial_{x_{i+v}}\left(\varphi \circ \Phi_{\varepsilon}\right) d \mu=\int_{X} \partial_{x_{i+v}} \tilde{\varphi}_{v} d \mu-\int_{X} \partial_{x_{i+v}} \varphi_{v} d \mu \leq|\varphi|_{\infty}\|\mu\|
$$

Observing equation (3.1), this yields

$$
\left\|\Phi_{\varepsilon}^{*} \mu\right\| \leq(2+2 d)\|\mu\|+C \ell_{\varepsilon}^{-1}|\mu| .
$$

This proves the second inequality of the lemma. The first one is trivial.

Proof of Lemma 2.4. The first two inequalities of this lemma are proved exactly as in Lemma 2.3. We turn to the third one. Let $A_{\varepsilon}=\cup_{v \in V} A_{\varepsilon, v}$ and notice that $\Phi_{\varepsilon}(x)=\Phi_{\varepsilon, i}(x)$ for all $x \in X$ such that $x_{i} \notin A_{\varepsilon}$. Thus, for all smooth local functions $\varphi$ and all $\mu \in \mathcal{B}$

$$
\left|\Phi_{\varepsilon}^{*} \mu(\varphi)-\Phi_{\varepsilon, i}^{*} \mu(\varphi)\right|=\sum_{v \in V}\left|\int_{X} 1_{A_{\varepsilon, v}}\left(x_{i}\right) 1_{A_{\varepsilon, v}}\left(x_{i+v}\right)\left[\varphi \circ \Phi_{\varepsilon}(x)-\varphi \circ \Phi_{\varepsilon, i}(x)\right] \mu(d x)\right|
$$

Next, since $\varphi \circ \Phi_{\varepsilon}$ depends only on finitely many variables, say the variables in the finite set $\Lambda_{0} \subset \mathbb{Z}^{d}$, we can consider the marginal of $\mu$ on $[0,1]^{\Lambda}, \Lambda=\Lambda_{0} \cup$ $\{i+v\}_{v \in V \cup\{0\}}$. Such a marginal is absolutely continuous with respect to Lebesgue measure, and its density $h$ is a function of bounded variation with $|h|_{B V} \leq\|\mu\|$, (see [7] for details). Hence,

$$
\left|\Phi_{\varepsilon}^{*} \mu(\varphi)-\Phi_{\varepsilon, i}^{*} \mu(\varphi)\right| \leq \sum_{v \in V} 2|\varphi|_{\infty} \int_{[0,1]^{\Lambda}} 1_{A_{\varepsilon, v}}\left(x_{i}\right) 1_{A_{\varepsilon,-v}}\left(x_{i+v}\right)|h(x)| d x
$$


and, since $|(|h|)|_{B V} \leq|h|_{B V}$ (see e.g. the proof of Lemma 2.3 in [2]), we can consider the marginal $h_{v}\left(x_{i}, x_{i+v}\right):=\int|h(x)| d x_{k \notin\{i, i+v\}}$. As $\left|h_{v}\right|_{B V} \leq|(|h|)|_{B V} \leq$ $|h|_{B V} \leq\|\mu\|$, the usual Sobolev inequalities imply

$$
\begin{aligned}
\left|\Phi_{\varepsilon}^{*} \mu(\varphi)-\Phi_{\varepsilon, i}^{*} \mu(\varphi)\right| & \leq \sum_{v \in V} 2|\varphi|_{\infty} \int_{[0,1]^{2}} 1_{A_{\varepsilon, v}}(x) 1_{A_{\varepsilon,-v}}(y) h_{v}(x, y) d x d y \\
& \leq 2(2 d)|\varphi|_{\infty}\|\mu\| \varepsilon .
\end{aligned}
$$

\section{REFERENCES}

[1] Baladi V., Gouezel S., Good Banach spaces for piecewise hyperbolic maps via interpolation, arXiv:0711.1960v1

[2] Bardet J.-B., Gouëzel S. and Keller G., Limit theorems for coupled interval maps, Stochastics and Dynamics 7 (2007), 17-36

[3] Lectures from the school-forum (CML 2004) held in Paris, June 21-July 2, 2004. Edited by J.-R. Chazottes and B. Fernandez. Lecture Notes in Physics, 671. Springer, Berlin (2005).

[4] Demers M. and Liverani C., Stability of Statistical Properties in Two-dimensional Piecewise Hyperbolic Maps, Transactions of the American Mathematical Society 360 (2008), 4777-4814

[5] P. Gaspard and T. Gilbert, Heat Conduction and Fourier's Law by Consecutive Local Mixing and Thermalization Physical Review Letters 101020601 (2008)

[6] P. Gaspard and T. Gilbert, Heat conduction and Fourier's law in a class of many particle dispersing billiards, New Journal of Physics 10103004 (2008)

[7] Keller G. and Liverani C., A spectral gap for a one-dimensional lattice of coupled piecewise expanding interval maps, in: Dynamics of Coupled Map Lattices and of Related Spatially Extended Systems (Eds.: J.-R. Chazottes, B. Fernandez), Lecture Notes in Physics 671 (2005), pp. 115-151, Springer Verlag

[8] Keller G. and Liverani C., Uniqueness of the SRB measure for piecewise expanding weakly coupled map lattices in any dimension, Communications in Mathematical Physics, 262, 1, 33-50, (2006)

[9] L. Bunimovich, C.Liverani, S. Pellegrinotti and Y.Suhov, Ergodic Systems of $n$ Balls in a Billiard Table, Communications in Mathematical Physics, 146, pp. 357-396, (1992).

Gerhard Keller, Department Mathematik, Universität Erlangen-Nürnberg, BismarCKstr. $1 \frac{1}{2}, 91052$ Erlangen, Germany

E-mail address: keller@mi.uni-erlangen.de

Carlangelo Liverani, Dipartimento di Matematica, II Università di Roma (Tor Vergata), Via della Ricerca Scientifica, 00133 Roma, Italy.

E-mail address: liverani@mat.uniroma2.it 